\title{
Role of miR-449b-3p in endometriosis via effects on endometrial stromal cell proliferation and angiogenesis
}

\author{
YUKAI LIU ${ }^{1}$, JIE CHEN ${ }^{2}$, XIAOYONG ZHU $^{1}$, LINGLI TANG ${ }^{1}$, XUEZHEN LUO $^{1}$ and YINGLI SHI ${ }^{1}$ \\ ${ }^{1}$ Laboratory for Reproductive Immunology, Hospital and Institute of Obstetrics and Gynecology, \\ Shanghai Medical College, Fudan University, Shanghai 200011; \\ ${ }^{2}$ Child Health Care Hospital Affiliated to Nanjing Medical University, Nanjing, Jiangsu 210029, P.R. China
}

Received December 21, 2017; Accepted June 26, 2018

DOI: $10.3892 / \mathrm{mmr} .2018 .9341$

\begin{abstract}
Endometriosis is a common gynecological disease and the pathogenesis is not clearly understood. Previous studies have demonstrated via microarray techniques that microRNA (miR)-449b was significantly downregulated both in ectopic and eutopic endometrium in patients with endometriosis. In the present study, the aberrant expression of miR-449b was further confirmed by reverse transcription-quantitative polymerase chain reaction. It was demonstrated that miR-449b-3p was downregulated in ectopic and eutopic tissues from women with endometriosis, and the same expression pattern was observed in endometrial stromal cells (ESCs) of eutopic endometrium from women with endometriosis and normal endometrium from women without endometriosis. Functional analysis, including an MTT assay, apoptosis conducted by flow cytometry, capillary-like tube formation assay and invasion assay, indicated that the upregulated expression of miR-449b-3p inhibited the proliferation of ESCs and that the supernatants of miR-449b-overexpressing ESCs inhibited the formation of tubular structures in human umbilical vein endothelial cells, whereas it has no effect on ESC apoptosis and invasiveness. These results suggest that the aberrant expression of miR-449b-3p was involved in the development and progression of endometriosis.
\end{abstract}

\section{Introduction}

Endometriosis, one of the most prevalent causes of pelvic pain, infertility and menstrual disorders, is a common chronic gynecological disorder of women at reproductive age. Despite

Correspondence to: Professor Yingli Shi, Laboratory for Reproductive Immunology, Hospital and Institute of Obstetrics and Gynecology, Shanghai Medical College, Fudan University, 588 Fangxie Road, Shanghai 200011, P.R. China

E-mail: ylshi2005@163.com

Key words: microRNA-449b, endometriosis, endometrial stromal cells, pathogenesis, proliferation, angiogenesis intensive research efforts, there is still a lack of in-depth knowledge regarding the molecular basis of the disease.

MicroRNAs (miRs) are RNA transcripts 19-22 nucleotides in length. The mechanism of miRs has been extensively studied in various pathological conditions and the expression profiles of miRs in a number of diseases, including endometriosis, have also been investigated. A single miR can target multiple genes, resulting in the regulation of target mRNA expression $(1,2)$. Therefore, alterations in the dynamic balance between miRs and their target mRNAs may alter the normal physiological status of tissues and may initiate pathological processes. Emerging data indicate that aberrant miR expression is associated with endometriosis, possibly mediating the development and progression of endometriosis by modulating proliferation, apoptosis, migration, invasion and estradiol signal transduction in endometriotic cells (3-5).

It is well known that miR-449b can alter the expression of certain molecule associated with adhesion and invasion. Likewise, endometrial stromal cells from endometriosis patients also changed in these aspects. Therefore it was hypothesised that miR-449b serves an important role in the development of endometriosis. The present study aimed to identify the expression and function of miR-449b, a differentially expressed $\mathrm{miR}$ in ectopic and eutopic tissues. The present study was designed to evaluate the role of miR-449b in the pathogenesis of endometriosis. Using miR-449b-transfected endometrial stromal cells (ESCs), the functional properties of miR-449b were observed. The results from these experiments will help us to better understand the miR-449b-mediated molecular mechanisms in ESCs.

\section{Materials and methods}

Tissue acquisition. The present study was approved by the ethics committee of Obstetrics and Gynecology Hospital (Shanghai, China). Ectopic (endometrioma; $n=19$ ), eutopic $(n=19)$ and normal $(n=35)$ endometrial tissues from patients with or without endometriosis, respectively who had undergone the laparoscopy and uterine curettage were obtained at the Obstetrics and Gynecology Hospital, Fudan University (Shanghai, China) from June 2017 to September 2017. None of the patients had received any hormonal treatments for at least half a year prior to the operation. The menstrual cycle phases 
of the patients were all in the proliferative phases, as assessed by medical history and a histological evaluation of the endometrium with the assistance of pathologists. The average age of the patients in the normal group was 32.7 \pm 6.8 years and that of the endometriosis group was $34.6 \pm 5.2$ years. Patients consented to tissue donation prior to surgery. Each sample was divided into two parts for mRNA extraction and isolation.

Cell culture and treatment. ESCs from endometrium with or without endometriosis were cultured by enzymatic digestion with collagenase as previously described (6), the deposit was re-suspended in DMEM/F-12 (HyClone; GE Healthcare Life Sciences, Logan, UT, USA) that contained $10 \%$ fetal bovine serum (FBS), as well as $100 \mathrm{U} / \mathrm{ml}$ penicillin and $100 \mathrm{mg} / \mathrm{ml}$ streptomycin (both from Gibco; Thermo Fisher Scientific, Inc., Waltham, MA, USA). The ESCs were purified through cell passage. After two generations, the purity of ESCs can reach $>95 \%$, which had been determined by flow cytometry with Alexa Fluor 488 anti-human vimentin mAb (clone: RV202; BD Biosciences, Franklin Lakes, NJ, USA) according to the protocol of the manufacturer $(5 / 100 \mu \mathrm{l})$. Following serum starvation for $12 \mathrm{~h}, \mathrm{ESCs}$ without endometriosis $\left(1 \times 10^{5}\right.$ cells/well) were treated with progesterone $\left(\mathrm{P} ; 10^{-8} \mathrm{~mol} / \mathrm{l}\right), 17 \beta$-estradiol $\left(\mathrm{E}_{2} ; 10^{-8} \mathrm{~mol} / \mathrm{l}\right)$ or $\mathrm{E}_{2}\left(10^{-8} \mathrm{~mol} / \mathrm{l}\right)+\mathrm{P}\left(10^{-8} \mathrm{~mol} / \mathrm{l}\right)$ for $24 \mathrm{~h}$; vehicle controls were also assayed (treated with ethanol, $2 \times 10^{-5} \mathrm{~mol} / 1, \mathrm{E}_{2}$ solution).

Fluorescence-based reverse transcription-quantitative polymerase chain reaction (RT-qPCR). The TRIzol reagent (Takara Bio, Inc., Otsu, Japan) was used to isolate total RNA. Next, cDNA was synthesized and amplified using the SYBR ${ }^{\circledR}$ PrimeScript $^{\mathrm{TM}}$ RT Master Mix kit (Takara Bio, Inc.) and the ABI PRISM 500 Sequence Detection System (Applied Biosystems; Thermo Fisher Scientific, Inc.) according to the manufacturer's protocol. The thermocycling conditions for reverse transcription was as follows: $37^{\circ} \mathrm{C} 15 \mathrm{~min}, 85^{\circ} \mathrm{C} 5 \mathrm{sec}$, and $4^{\circ} \mathrm{C}$ for storage. The gene used for normalization was Hsa-U6 small nuclear RNA (snRNA). The primers used were as follows: 5'-CGCGCGTGAATTACCGAA G-3' (forward) and 5'-GTGCAGGGTCCGAGGT-3' (reverse) for miR-449b-3p; 5'-CGCGCTATGGCACTGGTAG-3' (forward) and 5'-GTGCAGGGTCCGAGGT-3' (reverse) for miR-449b-5p; 5'-GCGCGTCGTGAAGCGTTC-3' (forward) and 5'-GTGCAG GGTCCGAGGT-3' (reverse) for Hsa-U6 snRNA. The conditions for qPCR were determined according to the protocol of the SYBR-Green JumpStart Taq ReadyMix kit (Sigma-Aldrich; Merck KGaA, Darmstadt, Germany). qPCR was implemented on a 7300 Real-Time PCR Detection System (ABI). The incubation condition for qPCR was as follows: Stage $1\left(95^{\circ} \mathrm{C} 30 \mathrm{sec}\right)$; stage 2 (40 cycle, $\left.95^{\circ} \mathrm{C} 5 \mathrm{sec} ; 60^{\circ} \mathrm{C} 31 \mathrm{sec}\right)$; stage $3\left(95^{\circ} \mathrm{C} 15 \mathrm{sec} ; 60^{\circ} \mathrm{C}\right.$ $\left.1 \mathrm{~min} ; 95^{\circ} \mathrm{C} 15 \mathrm{sec}\right)$. The results were expressed as arbitrary units defined by the $2^{-\Delta \Delta C t}$ method (7).

miR-449b lentivirus construction and transduction. The precursor of the miR hsa-miR-449b-3p was constructed by Genechem Co., Ltd. (Shanghai, China). In the present study, the RNA primers used for the amplification of the target gene were as follows: 5'-GAGGATCCCCGGGTACCGGGTGAC TATTAAGATTAGAGTTCTG-3' and 5'-CACACATTCCAC AGGCTAGGACAGCAGTTGCATGTTAGC-3', which had been confirmed by sequencing. The control green fluorescence protein-lentivirus, (GFP-LV) and the recombinant lentivirus overexpressing miR-449b-3p (miR-449b-LV) were prepared and diluted to $1.0^{9}$ transfection $\mathrm{U} / \mathrm{ml}$. Preparation included the following four steps: Target gene insertion and plasmid construction, packaging processing, purification and amplification, dilution and storage, which had been conducted by Genechem Co., Ltd. (Shanghai, China) according to the protocol of the manufacturer (Genechem Co., Ltd.).

The ESCs of the normal group were plated in 6-well plates $\left(5 \times 10^{4}\right.$ cells/well) at $37^{\circ} \mathrm{C}$ under $5 \% \mathrm{CO}_{2}$ overnight. The next day, following discarding the supernatants, $0.2 \mathrm{ml}$ fresh complete medium containing lentiviruses and polybrene $(8 \mathrm{mg} / \mathrm{ml})$ was added to ESCs at $37^{\circ} \mathrm{C}$ under $5 \% \mathrm{CO}_{2}$ for $12 \mathrm{~h}$. Then ESCs were incubated in $0.3 \mathrm{ml}$ freshly prepared polybrene-Dulbecco's modified Eagle's medium (DMEM; HyClone, GE Healthcare Life Sciences) for another $24 \mathrm{~h}$. Finally, following discarding the supernatants and replacing with fresh DMEM, the cells were cultured for 3 days. The efficiency of lentivirus transduction was investigated by the detection of GFP signals using fluorescence microscopy (IX71; Olympus Corporation, Tokyo, Japan) at $72 \mathrm{~h}$ following transduction. The expression of miR-449b-3p in stably transduced ESCs was tested by RT-qPCR. The ESCs transduced with miR-449b-LV (miR-449b up) and GFP-LV (NC) were cryopreserved for further functional analysis.

Measurement of cell viability by MTT assay. The ESCs (control/untransfected cells/NC/miR-449bup) (2.0x10 ${ }^{3}$ cells/well) were cultured in DMEM supplemented with $10 \%$ fetal bovine serum (FBS; Gibco; Thermo Fisher Scientific, Inc.) in 96-well plates (Costar; Corning, Inc., Corning, NY, USA). Following incubation for 1-5 days, $10 \mu \mathrm{l}$ MTT (Sigma-Aldrich; Merck $\mathrm{KGaA}$ ) solution $\left(5 \mathrm{mg} / \mathrm{ml}\right.$ in $\left.\mathrm{dd}_{2} \mathrm{O}\right)$ was added to the wells. The plates were incubated at $37^{\circ} \mathrm{C}$ for $4 \mathrm{~h}$. Intracellular formazan crystals were dissolved by adding $100 \mu \mathrm{l}$ DMSO to each well. Cell proliferation was evaluated on a microplate reader (BioTek Instruments, Inc., Winooski, VT, USA) set to $490 \mathrm{~nm}$.

Measurement of apoptosis by flow cytometry. According to the protocol of the BD Annexin V Staining kit (BD Biosciences, Franklin Lakes, NJ, USA), the apoptosis assay was performed as previously described (8). Briefly, the ESCs (control/NC/miR-449b up) were trypsinized and collected at a concentration of $1 \times 10^{6}$ cells $/ \mathrm{ml}$. Following incubation in allophycocyanin-Annexin $\mathrm{V}$ ( $5 \mu \mathrm{l} /$ test tube respectively) for $15 \mathrm{~min}$ at room temperature in the dark, the cells were tested by flow cytometry (Beckman Coulter, Inc., Brea, CA, USA) as soon as possible (within $1 \mathrm{~h}$ ). The experimental results were analyzed using FlowJo software (X10.0.7; BD Biosciences).

Measurement of angiogenesis by capillary-like tube formation assay. The ESCs transfected with miR-449b-LV, GFP-LV and normal ESCs $\left(2.0 \times 10^{5}\right.$ cells/well) were seeded in 6-well plates. Following $24 \mathrm{~h}$ incubation, the supernatants were transferred into $15 \mathrm{ml}$ centrifuge tubes and centrifuged at $500 \mathrm{x}$ g at $4^{\circ} \mathrm{C}$ for $5 \mathrm{~min}$. Then, the supernatants were collected for further experiments. Human umbilical vein endothelial cells (HUVECs; American Type Culture Collection, Manassas, VA, USA; $2.0 \times 10^{4}$ cells/well) were seeded on a thin layer of Matrigel (BD Biosciences) that had been incubated at $37^{\circ} \mathrm{C}$ 

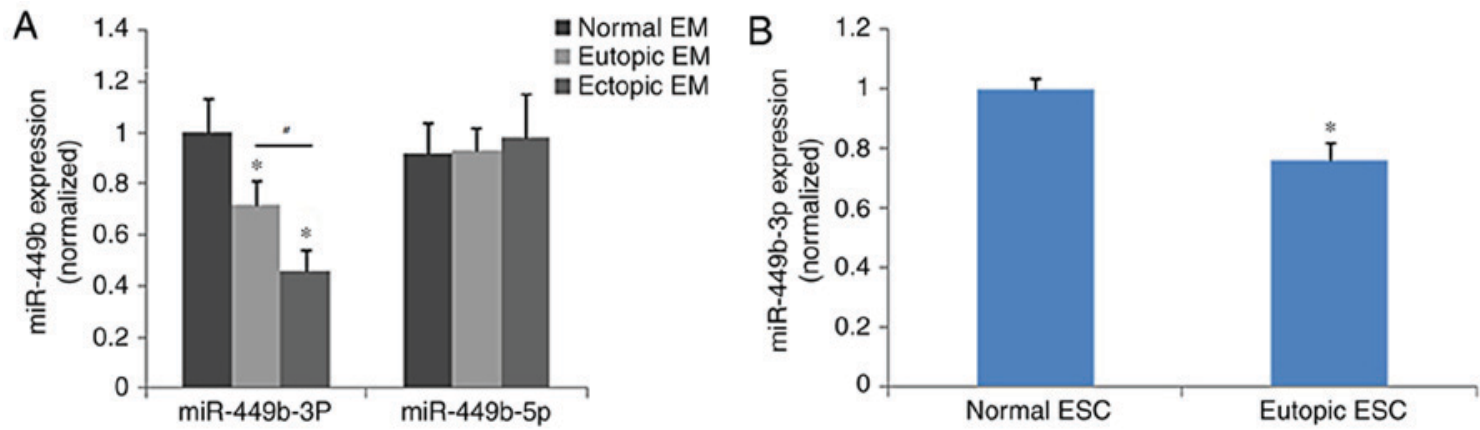

Figure 1. Expression of miR-449b was downregulated in endometriosis. (A) Expression of miR-449b-3p and miR-449b-5p in endometrial tissues was tested by RT-qPCR. Ectopic and eutopic endometrium from women with endometriosis $(n=19)$ and control endometrium from endometriosis-free women $(n=21)$. Values are presented as the mean \pm standard deviation, ${ }^{*} \mathrm{P}<0.05$ vs. normal EM (one-way analysis of variance). ${ }^{\#} \mathrm{P}<0.05$. Bonferroni's multiple comparisons test was used for the post-hoc test to compare specific groups. (B) Expression of miR-449b-3p in ESCs was tested by RT-qPCR. miR-449b-3p expression data are presented as the fold-change compared with the normal group. Values indicate the mean \pm standard deviation. Normal ESCs, $\mathrm{n}=21$; eutopic ESCs, $\mathrm{n}=19 ;{ }^{*} \mathrm{P}<0.05$ vs. normal ESC (two-tailed, unpaired t-test). EM, endometrium; RT-qPCR, reverse transcription-quantitative polymerase chain reaction; miR, microRNA; ESCs, endometrial stromal cells.

for $2 \mathrm{~h}$ in 24-well plates and cultured in RPMI-1640 medium (HyClone; GE Healthcare Life Sciences) supplemented with $1 \%$ FBS containing the abovementioned supernatants (normal $\mathrm{ESCs} / \mathrm{NC} / \mathrm{miR}-449 \mathrm{~b}$ up) for $6 \mathrm{~h}$ at $37^{\circ} \mathrm{C}$. Following fixation/permeabilization buffer (BD Biosciences) was added at $4^{\circ} \mathrm{C}$ for $15 \mathrm{~min}$ in accordance with the protocol of the manufacturer $\left(250 \mu \mathrm{l} / 10^{6}\right.$ cells $)$ and DAPI Staining Solution at $4^{\circ} \mathrm{C}$ for another $10 \mathrm{~min}$. The cell plates were scanned by Cellomics (Thermo Fisher Scientific, Inc.; ArrayScan VT1) and the data were analyzed with Cellomics software (6.1.0). Tube area, mean tube length and mean tube nodes were calculated automatically by the software, to measure the capillary-like structures. For rigor, three independent experiments were performed in triplicate.

Measurement of invasiveness by invasion (Matrigel) chamber assay. The ESCs (normal group/NC/miR-449b up; $2.5 \times 10^{4}$ ) were seeded on a cell culture Transwell insert that had been coated with extracellular matrix (ECM; 8-mm pore size, 24-well format; Costar; Corning, Inc.) in 2\% FBS medium. The complete medium (containing $10 \% \mathrm{FBS}$ ) was added into the lower chamber. ESCs were incubated at $37^{\circ} \mathrm{C}$ under $5 \% \mathrm{CO}_{2}$ for $24 \mathrm{~h}$ and then scratched from the upper chamber using a cotton swab. Next, the invaded cells were stained on the underside of the insert at room temperature with Giemsa staining solution. Following rinsing with PBS, images of the undersides of the membrane were captured using a light microscope to compare the number of invaded cells per insert. The invaded cells were scored by randomly counting 10 high-power fields per filter. The counting accuracy was validated by optical density $570 \mathrm{~nm}$ quantification of the methanol-solubilized dye, which was tested by a microplate reader (BioTek Instruments, Inc., Winooski, VT, USA).

Statistical analysis. The data represented the mean \pm standard deviation of at least 3 independent experiments. Data were analyzed using GraphPad Prism 6 (GraphPad Software Inc., La Jolla, CA, USA). The difference between two means was examined using a Student's t-test, while comparison of three or more groups was determined with one-way analysis of variance. $\mathrm{P}<0.05$ was considered to indicate a statistically significant difference. If $\mathrm{P}<0.05$, Bonferroni's multiple comparisons test was used for the post-hoc test.

\section{Results}

Quantification of miR-449b expression. The eutopic endometrium expressed significantly less miR-449b-3p compared with the control endometrium $(\mathrm{P}<0.05)$, and miR-449b-3p was significantly lower in samples of ectopic endometrium than in eutopic endometrium $(\mathrm{P}<0.05)$, whereas miR-449b-5p did not demonstrate significantly different expression among the groups (Fig. 1A). The expression of miR-449b in ESCs isolated from eutopic and normal endometrium was further tested as ESCs are involved in adhesion of endometrial tissues to the peritoneal lining in the early stages of endometriosis. The results of cell analysis were in accordance with those of the tissue samples $(\mathrm{P}<0.05$; Fig. 1B). The treatment of ESCs with ovarian steroids (17 $\beta$-estradiol and progesterone) did not regulate the expression of miR-449b-3p in ESCs ( $\mathrm{P}>0.05$; data not shown).

Transfection efficiency of recombinant lentivirus. To further investigate the roles of miR-449b-3p in ESCs, a lentiviral construct experiment for the overexpression of miR-449b-3p was prepared. In transfected cells, $>80$ percent of the cells exhibited GFP expression and maintained morphological features similar to those of untransfected cells (Fig. 2A). The percentages were estimated according to the intensity of green fluorescence by flow cytometry. RT-qPCR revealed that the transfection of ESCs with miR-449b-LV significantly increased miR-449b expression by 117 -fold ( $\mathrm{P}<0.0001$; Fig. 2B).

miR-449b induces proliferation, with no effect on apoptosis of ESCs. MTT assays were conducted to assess the effect of miR-449b-3p on cell proliferation. miR-449b-3p significantly suppressed proliferation of ESCs from day 4-5 compared with the negative control $(\mathrm{P}<0.05$; Fig. 3A).

To study whether miR-449b induced apoptosis in ESCs, an Annexin $\mathrm{V}$ assay was performed to determine cell apoptosis in miR-449b-overexpressing cells, the negative control group and the control group. There was no difference between the NC and miR-449b up group (4.3 vs. $4.14 \%$; P>0.05; Fig. 3B and C). 
A

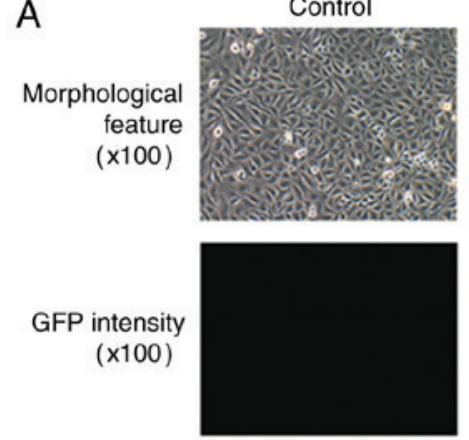

NC

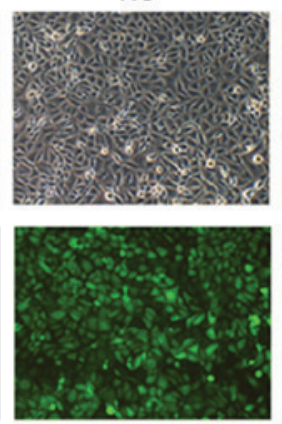

miR-449b up

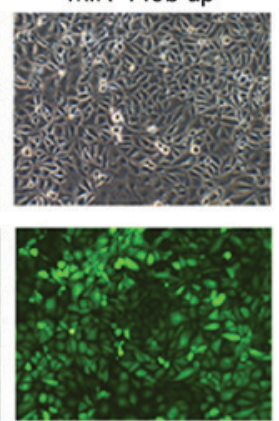

B

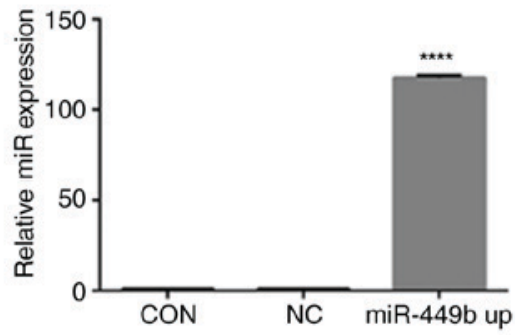

Figure 2. The infection of miR-449b-3p lentivirus into ESCs and its efficiency validation. (A) Cell morphological alterations were observed by inverted phase contrast microscopy (upper) and the transduction efficiency was determined by fluorescence microscopy (lower) following transduction with the recombinant lentivirus. Representative images are presented. (B) The expression of miR-449b-3p was tested by reverse transcription-quantitative polymerase chain reaction. Values indicate the mean \pm standard deviation. $\mathrm{n}=6,{ }^{* * * *} \mathrm{P}<0.0001$, vs. NC. Significance was assessed using a one-way analysis of variance. CON, control group ESCs; NC, negative control GFP-lentivirus infected ESCs; miR-449b up, miR-449b-3p lentivirus infected ESCs; ESCs, endometrial stromal cells; miR, microRNA.

This suggested that miR-449b may not directly have an apoptotic effect on ESCs.

miR-449b exhibits no effect on the invasive ability of ESCs. The effects of miR-449b-3p overexpression in ESCs were also examined using an invasion chamber that had been coated with ECM-Matrigel. There was no significant difference in numbers of cells passing through the matrix between the miR-449b-overexpressing group and the negative control group ( $\mathrm{P}>0.05$; Fig. 4).

Culture supernatants from miR-449b-3p-overexpressing ESCs enhance in vitro angiogenesis of HUVECs. To evaluate the effect of miR-449b-3p on the angiogenesis of HUVECs, an in vitro angiogenesis model was established. HUVECs were seeded on a thin layer of Matrigel that had solidified for $2 \mathrm{~h}$ and were incubated with the supernatants derived from the control group ESCs, NC ESCs and miR-449b-overexpressing ESCs, followed by a capillary-like tube formation assay. At $6 \mathrm{~h}$ following seeding, there was reduced formation of tubular structures in HUVECs treated with supernatants from miR-449b-overexpressing ESCs compared with supernatants from the NC group (Fig. 5A). Quantitative analysis revealed that compared with GFP-LV group, supernatants from the miR-449b-overexpressing ESCs inhibited the tube area (964 $37.33 \pm 1043.03$ vs. $\left.87077.00 \pm 2940.59 \mu \mathrm{m}^{2} ; \mathrm{P}=0.007\right)$, mean tube length $(490.25 \pm 53.12$ vs. $322.14 \pm 85.08 \mu \mathrm{m} ; \mathrm{P}=0.044)$ and mean tube node $(14.61 \pm 4.23$ vs. $6.21 \pm 1.33$; $\mathrm{P}=0.03$; Fig. $5 \mathrm{~B}-\mathrm{D})$.

\section{Discussion}

miR-449b has been implicated in several malignant, inflammatory and premature ovarian insufficiencies (9-11); however, its association with the pathogenesis of endometriosis has not previously been well described. It is well established that ectopic endometrium may have a better capacity to survive outside the uterine cavity because of its different functions compared with those of normal endometrium in women without endometriosis. Both genetic and acquired molecular abnormalities may alter the ectopic viability of the endometrium, potentially rendering certain women susceptible to endometriosis. The findings of the current study indicated that
miR-449b-3p was in ectopic and eutopic tissues, in accordance with the results of a previous study (12).

The cellular composition of ectopic tissues is heterogeneous and contains cells from surrounding ovarian tissue, inflammatory cells, endometrial stromal and epithelial cells in variable proportions. In fact, ectopic tissues may contain only a small fraction of endometrium-specific cells. Therefore, the heterogeneity of endometriotic lesion biopsies presents a real challenge in the study of endometriosis, as the molecular signature of endometrial cells in lesions could be masked by the surrounding tissue, leading to inconsistent or wrongly interpreted results (13). To overcome this issue, the differences in miR-449b levels were examined, focusing on the isolation and analysis of ESC. In the present study, it was demonstrated that miR-449b-3p expression in eutopic ESCs was decreased compared with the control group.

The expression levels of miRs generated either from $-5 p$ or $-3 p$ arms of the precursor may vary not only among various tissues/cells but also in various states of health and disease (14). miRs can act as regulators of the steroid hormone response in the female reproductive tract (15); conversely, a number miRs may also be affected by hormone levels (16). To confirm whether ovarian steroids have regulatory effects on miR-449b, miR-449b-3p expression in ESCs was measured following treatment with $17 \beta$-estradiol and progesterone by qPCR. However, in the present study, estrogen and progesterone have no effect on expression of miR-449b-3p.

The attachment and invasion of endometrium fragments is considered to be necessary for the formation of endometriosis. Simultaneously, the establishment of a blood supply and a suboptimal immune response provide favorable conditions for the development of endometriosis. Therefore, a number of relevant functional effects of ESCs were analyzed using in vitro assays.

In previous studies, miR-449b was upregulated in prostate cancer and $\mathrm{T}$ cells of patients with systemic lupus erythematosus, while it was downregulated in thyroid carcinoma and ovarian cancer, suggesting that its roles can vary according to the cellular context (17-20). It is involved in a number of cellular functions, including cell cycle control and cell differentiation (21). The induction of miR-449 expression can lead to cell cycle stagnation and apoptosis by inhibiting cyclin 

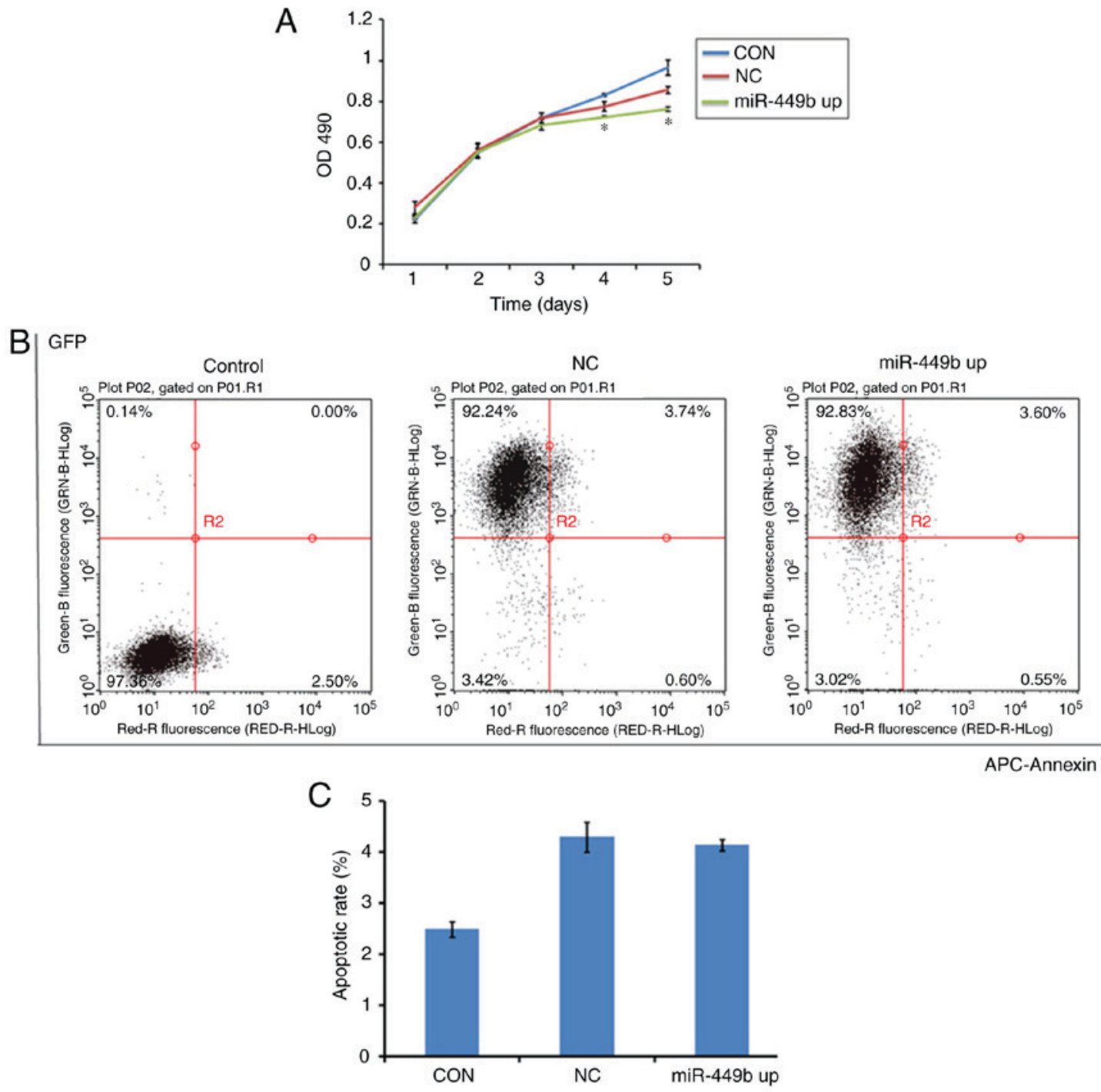

APC-Annexin V

Figure 3. Overexpression of miR-449b-3p facilitated proliferation of ESC, with no effect on apoptosis. (A) Proliferation of ESCs was measured by MTT assay. (B) Apoptosis of ESCs was tested by flow cytometry with an APC-Annexin V kit. Numbers in quadrants indicate the percentage of cells in different conditions. (C) Quantifications of the percentages of apoptosis are presented. Values indicate the mean \pm standard deviation, $n=6$. ${ }^{*} \mathrm{P}<0.05$, vs. NC (one-way analysis of variance). OD, optical density; CON, control group ESCs; NC, negative control GFP-lentivirus infected ESCs; miR-449b up, miR-449b-3p lentivirus infected ESCs; miR, microRNA; ESCs, endometrial stromal cells; GFP, green fluorescence protein; APC, allophycocyanin.

dependent kinase and cell division cycle 25A. It can also protect against the proliferation induced by E2F transcription factor 1 as a negative feedback mechanism (22). The present study demonstrated the effect of miR-449b on cell growth is via the modulation of cell proliferation rather than via apoptosis.

The further functional analysis in the present study indicates that miR-449b-3p serves an inhibitory role in promoting tubulogenesis of HUVECs, whereas it has no effect on cell invasiveness. Similar to tumors, the survival and growth of endometrium requires a blood supply. It has been demonstrated that eutopic endometrium from patients with endometriosis exhibits increased angiogenic potential in comparison with disease-free women, potentially contributing to the initiation of endometriosis (23).

The study of miR-449b-3p downstream mechanisms will be investigated further. In the present study, the abnormal expression of miR-449b-3p in endometriosis was clarified and the biological functional alterations brought about by the downregulation of miR-449b-3p were further investigated. Presently, the present study group is also trying to identify the downstream molecular targets of miR-449b-3p and hope to further explain the specific molecular mechanisms of miR-449b-3p.

In conclusion, it was demonstrated that miR-449b-3p was downregulated in ectopic and eutopic tissues, and the same expression pattern was also observed in ESCs. Its expression is not affected by estrogen or progesterone. The upregulated expression of miR-449b-3p inhibited the proliferation of ESCs and the supernatants of miR-449b-overexpressing ESCs inhibited the formation of tubular structures in HUVECs. The present study group is still investigating targets of this miR that are associated with cellular functions. The impact of miR- $449 \mathrm{~b}$ on endometriosis in vivo can be expected to improve the implantation and establishment of ectopic lesions. These results suggest that abnormalities in miR-449b expression lead to the development and progression of endometriosis. 

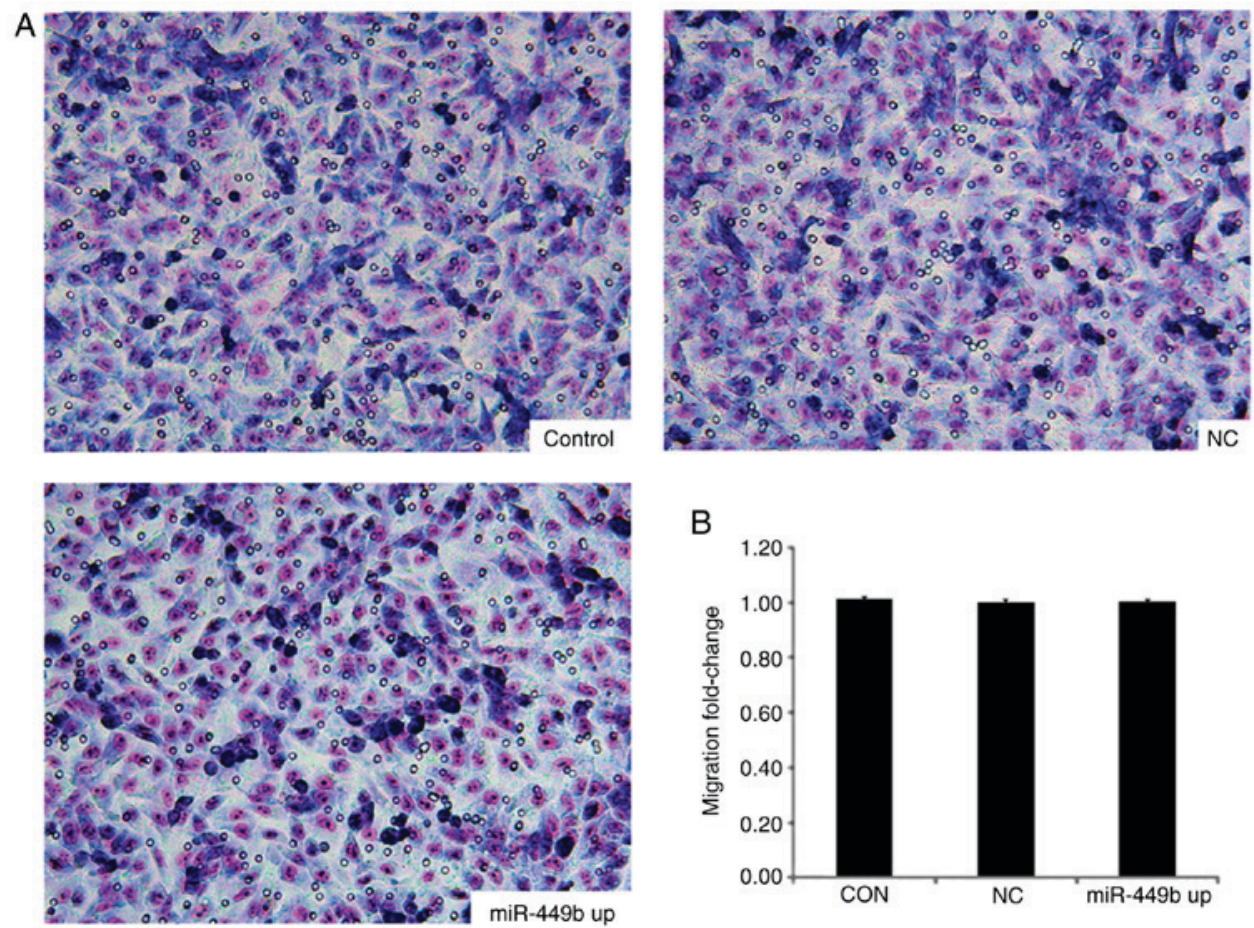

Figure 4. Overexpression of miR-449b-3p had no effect on the invasiveness of ESCs. (A) Invasiveness of ESCs was observed by a light microscope. The invaded cells were scored by randomly counting 10 high-power fields per filter. Representative images are presented. (B) Quantifications of the invasive cells. Values indicate the mean \pm standard deviation, $\mathrm{n}=6$, significance was assessed using a one-way analysis of variance. CON, control group ESCs; $\mathrm{NC}$, negative control lentivirus infected ESCs; miR-449b up, miR-449b-3p lentivirus infected ESCs; miR, microRNA; ESCs, endometrial stromal cells.

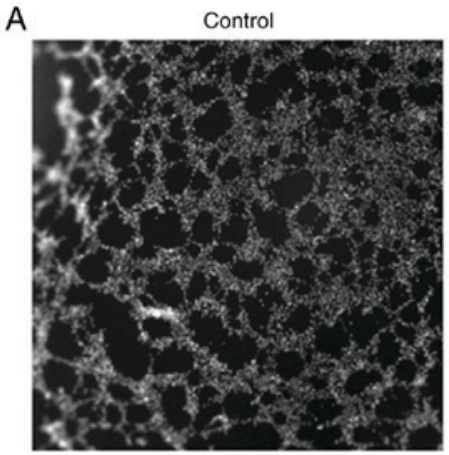

B

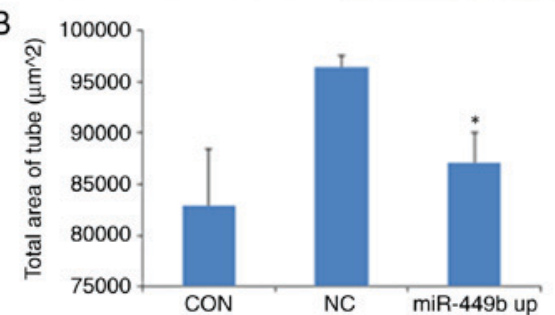

NC

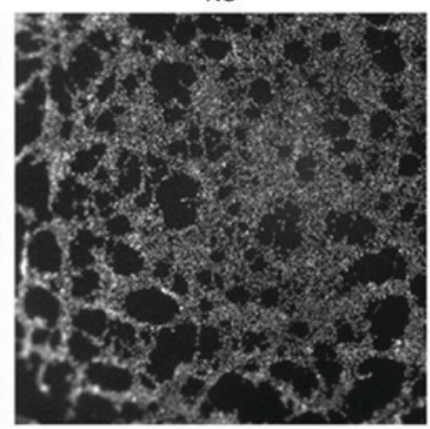

miR-449b up
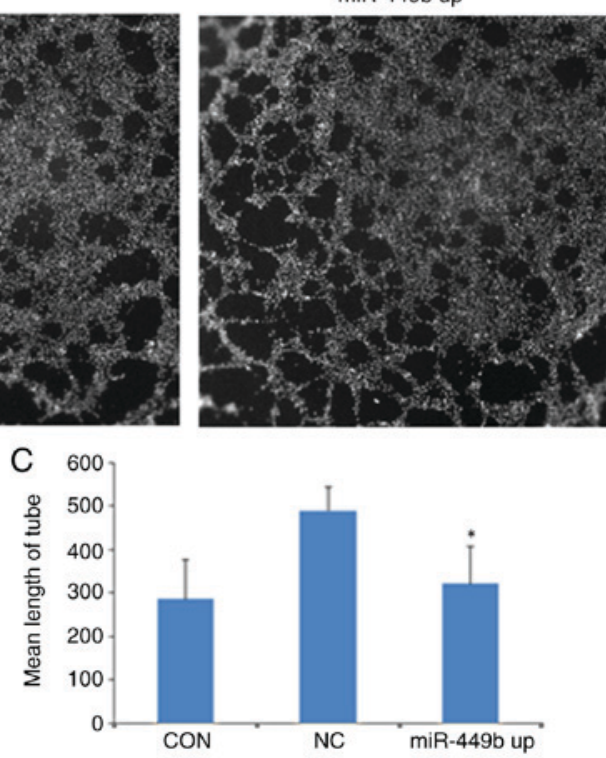

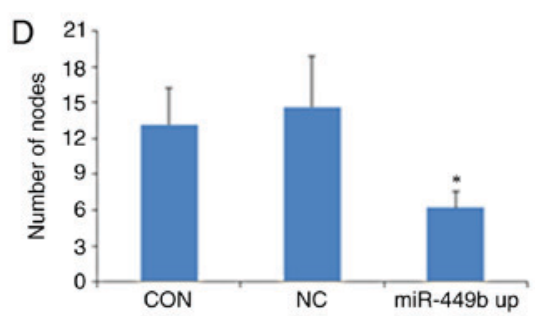

Figure 5. The supernatant of miR-449b-3p-overexpressing ESCs reduced the angiogenetic capacity of human umbilical vein endothelial cells. (A) Capillary-like tube formation was measured by a Cellomics instrument. Representative images are presented. (B) Tube area, (C) mean tube length and (D) mean tube nodes were acquired automatically by Cellomics analysis software to measure the capillary-like structures. Values indicate the mean \pm standard deviation, $\mathrm{n}=6$. ${ }^{*} \mathrm{P}<0.05$, significance was assessed using a one-way analysis of variance. CON, supernatant of control group ESCs; NC, supernatant of negative control lentivirus infected ESCs; miR-449b up, supernatant of miR-449b-3p lentivirus infected ESCs; miR, microRNA; ESCs, endometrial stromal cells. 


\section{Acknowledgements}

Not applicable.

\section{Funding}

This study was supported by funds from the National Natural Science Foundation of China (grant no. 81471438).

\section{Availability of data and materials}

The datasets used and/or analyzed during the current study are available from the corresponding author on reasonable request.

\section{Authors' contributions}

YL conducted all the experiments and arranged the figures and the manuscript. XZ, LT and XZ assisted with sample collection. JC assisted with analyzing the data and revising the manuscript critically. YS initiated and supervised the project, analyzed the data and formed the conclusion, and edited the manuscript.

\section{Ethics approval and consent to participate}

The present study was approved by the ethics committee of Obstetrics and Gynecology Hospital and patients consented to tissue donation prior to surgery. Written informed consent was obtained from all patients.

\section{Patient consent for publication}

Not applicable.

\section{Competing interests}

The authors declare that they have no competing interests.

\section{References}

1. Pillai RS: MicroRNA function: Multiple mechanisms for a tiny RNA? RNA 11: 1753-1761, 2005.

2. Engels BM and Hutvagner G: Principles and effects of microRNA-mediated post-transcriptional gene regulation. Oncogene 25: 6163-6169, 2006.

3. Nematian SE, Mamillapalli R, Kadakia TS, Majidi Zolbin M, Moustafa S and Taylor HS: Systemic inflammation induced by microRNAs: Endometriosis derived alterations in circulating microRNA $125 b-5 p$ and Let $7 b-5 p$ regulate macrophage cytokine production. J Clin Endocrinol Metab 103: 64-74, 2018.

4. Park JH, Lee SK, Kim MK, Lee JH, Yun BH, Park JH, Seo SK Cho S and Choi YS: Saponin extracts induced apoptosis of endometrial cells from women with endometriosis through modulation of miR-21-5p. Reprod Sci 25: 292-301, 2018.

5. Joshi NR, Miyadahira EH, Afshar Y, Jeong JW, Young SL, Lessey BA, Serafini PC and Fazleabas AT: Progesterone resistance in endometriosis is modulated by the altered expression of MicroRNA-29c and FKBP4. J Clin Endocrinol Metab 102: 141-149, 2017.
6. Shi YL, Luo XZ, Zhu XY, Hua KQ, Zhu Y and Li DJ: Effects of combined 17beta-estradiol with TCDD on secretion of chemokine IL-8 and expression of its receptor CXCR1 in endometriotic focus-associated cells in co-culture. Hum Reprod 21: 870-879, 2006.

7. Livak KJ and Schmittgen TD: Analysis of relative gene expression data using real-time quantitative PCR and the 2(-Delta Delta C(T)) method. Methods 25: 402-408, 2001.

8. Shi XY, Gu L, Chen J, Guo XR and Shi YL: Downregulation of miR-183 inhibits apoptosis and enhances the invasive potential of endometrial stromal cells in endometriosis. Int J Mol Med 33: 59-67, 2014.

9. Sandbothe M, Buurman R, Reich N, Greiwe L, Vajen B, Gürlevik E, Schäffer V, Eilers M, Kühnel F, Vaquero A, et al: The microRNA-449 family inhibits TGF- $\beta$-mediated liver cancer cell migration by targeting SOX4. J Hepatol 66: 1012-1021, 2017.

10. Pan H, Chen B, Wang J, Wang X, Hu P, Wu S, Liu Y, Xu Z, Zhang W, Wang B and Cao Y: The miR-449b polymorphism, rs10061133 A>G, is associated with premature ovarian insufficiency. Menopause 23: 1009-1011, 2016.

11. Buggele WA, Krause KE and Horvath CM: Small RNA profiling of influenza A virus-infected cells identifies miR-449b as a regulator of histone deacetylase 1 and interferon beta. PLoS One 8: e76560, 2013.

12. Braza-Boïls A,Marí-Alexandre J,GilabertJ,Sánchez-IzquierdoD, España F, Estellés A and Gilabert-Estellés J: MicroRNA expression profile in endometriosis: Its relation to angiogenesis and fibrinolytic factors. Hum Reprod 29: 978-988, 2014.

13. Saare M, Rekker K, Laisk-Podar T, Rahmioglu N, Zondervan K, Salumets A, Götte M and Peters M: Challenges in endometriosis miRNA studies-From tissue heterogeneity to disease specific miRNAs. Biochim Biophys Acta 1863: 2282-2292, 2017.

14. Meijer HA, Smith EM and Bushell M: Regulation of miRNA strand selection: Follow the leader? Biochem Soc Trans 42: 1135-1140, 2014.

15. Sørensen AE, Udesen PB, Wissing ML, Englund AL and Dalgaard LT: MicroRNAs related to androgen metabolism and polycystic ovary syndrome. Chem Biol Interact 259: 8-16, 2016.

16. Lam EW, Shah K and Brosens JJ: The diversity of sex steroid action: The role of micro-RNAs and FOXO transcription factors in cycling endometrium and cancer. J Endocrinol 212: 13-25, 2012.

17. Mortensen MM, Høyer S, Orntoft TF, Sørensen KD, Dyrskjøt L and Borre M: High miR-449b expression in prostate cancer is associated with biochemical recurrence after radical prostatectomy. BMC Cancer 14: 859, 2014.

18. Lu MC Yu CL, Chen HC, Yu HC, Huang HB and Lai NS: Aberrant $\mathrm{T}$ cell expression of $\mathrm{C}^{\mathrm{a} 2+}$ influx-regulated miRNAs in patients with systemic lupus erythematosus promotes lupus pathogenesis. Rheumatology (Oxford) 54: 343-348, 2015.

19. Chen L, Xu L and Wang G: Regulation of MET-mediated proliferation of thyroid carcinoma cells by miR-449b. Tumour Biol 36: 8653-8660, 2015.

20. Ma Lp, Li N, He Xj and Zhang Q: miR-449b and miR-34c on inducing down-regulation of cell cycle-related proteins and cycle arrests in SKOV3-ipl cell, an ovarian cancer cell line. Beijing Da Xue Xue Bao 43: 129-133, 2011 (In Chinese).

21. Fang Y, Gu X, Li Z, Xiang J and Chen Z: miR-449b inhibits the proliferation of SW1116 colon cancer stem cells through downregulation of CCND1 and E2F3 expression. Oncol Rep 30: 399-406, 2013

22. Yang X,Feng M, Jiang X, Wu Z, Li Z, Aau M and Yu Q: miR-449a and miR-449b are direct transcriptional targets of E2F1 and negatively regulate $\mathrm{pRb}-\mathrm{E} 2 \mathrm{~F} 1$ activity through a feedback loop by targeting CDK6 and CDC25A. Genes Dev 23: 2388-93, 2009.

23. Laschke MW and Menger MD: Anti-angiogenic treatment strategies for the therapy of endometriosis. Hum Reprod Update 18: 682-702, 2012.

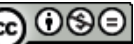

This work is licensed under a Creative Commons Attribution-NonCommercial-NoDerivatives 4.0 International (CC BY-NC-ND 4.0) License. 